\title{
Acute oropharyngeal palsy is associated with antibodies to GQ1b and GT1a gangliosides
}

\author{
C P O'Leary, J Veitch, W F Durward, A M Thomas, J H Rees, H J Willison
}

\begin{abstract}
Three patients with acute oropharyngeal palsy had high titre anti-GQ1b and antiGT1a IgG antibodies. No patients had ophthalmoplegia or ptosis. In all patients limb ataxia or areflexia were present without notable limb weakness. These patients describe an oropharyngeal variant of Guillain-Barré syndrome in terms of anti-GQ1b antibody reactivity and show that high titre anti-GQ1b antibodies, serologically indistinguishable from those found in Miller Fisher syndrome, can occur in a clinical setting without ophthalmoplegia. The anti-GQ1b and anti-GT1a antibody assays may be helpful tests when considering the differential diagnosis of acute oropharyngeal palsy.
\end{abstract}

(F Neurol Neurosurg Psychiatry 1996;61:649-651)

Keywords: Miller Fisher syndrome; oropharyngeal palsy; Guillain-Barré syndrome; gangliosides

Several regional variants of Guillain-Barré syndrome have been described occurring either as isolated syndromes or as transitional illnesses that either overlap each other or evolve into generalised Guillain-Barré syndrome. ${ }^{1}$ Of these, the most widely recognised is the Miller Fisher syndrome, ${ }^{2}$ in which serum antibodies to GQ1b are found in over $90 \%$ of patients. ${ }^{3-5}$ Anti-GQ1b antibodies are also present in patients with Guillain-Barré syndrome who exhibit ophthalmoplegia. ${ }^{67}$ These clinicoserological studies have led some authors to suggest that anti-GQ1b antibodies are a marker for ophthalmoplegia. This conclusion is also supported by the finding that GQ1b is highly enriched in human oculomotor nerves compared with peripheral nerve. ${ }^{7}$

Acute oropharyngeal palsy is another regional variant of Guillain-Barré syndrome. It may occur in isolation, as part of the Miller Fisher syndrome, or with mild proximal upper limb weakness, when it has been referred to as the pharyngeal-cervical-brachial variant, comprising $2 \%$ of patients with Guillain-Barré syndrome. ${ }^{1}$ One such patient, transitional with generalised Guillain-Barré syndrome, has been reported in association with anti-GT1a and GD1a antibodies. ${ }^{8}$ Acute oropharyngeal palsy can also occur with ataxia and tendon reflex abnormalities, as described here. When ophthalmoplegia completes the triad these patients are usually referred to as having Miller Fisher syndrome.

Here we report three patients with acute oropharyngeal palsy without ophthalmoplegia, in whom high titre anti-GQ1b and GT1a antibodies were detected. This clinicoserological association has not previously been reported and shows a further regional variant of Guillain-Barré syndrome with a readily identifiable antiganglioside antibody phenotype.

\section{Case reports and assay methods}

Table 1 summarises the clinical features of the three patients. The initial symptoms in all patients were of dysarthria and dysphagia. At no time did any patient complain of double vision or have clinically evident ophthalmoplegia. In each patient there was no significant functional involvement of other sites although minor symptoms and signs, including paraesthesiae, limb weakness, ataxia, and areflexia were variably present. One patient required tube feeding and two required dietary modification to tolerate an oral intake. All patients made a good recovery. The preceding infections were unidentified upper respiratory tract infections in two patients and serologically established Campylobacter jejuni enteritis in the other patient. Peripheral nerve electrophysiology was abnormal in all patients showing both motor and sensory nerve conduction abnormalities.

A control group of 10 consecutive patients with Miller Fisher syndrome, with the triad of ophthalmoplegia, ataxia, and areflexia, was studied for comparison (summarised in table 1). Nine of these patients with Miller Fisher syndrome had craniopharyngeal weakness without notable limb weakness, this being the typical pattern of Miller Fisher syndrome seen locally. All patients had preceding unidentified upper respiratory tract infections and abnormal electrophysiological studies compatible with the diagnosis of Miller Fisher syndrome.

Serum samples were collected four days (patient 1), 14 days (patient 2), and eight days (patient 3) after the onset of symptoms and before specific treatment was started. Samples were stored at $-70^{\circ} \mathrm{C}$ until assayed. Antibody 
Table 1 Clinical features of the three oropharyngeal patients and the 10 Miller Fisher (MFS) patients

\begin{tabular}{|c|c|c|c|c|}
\hline & Patient 1 & Patient 2 & Patient 3 & $10 \mathrm{MFS}$ patients \\
\hline \multicolumn{5}{|l|}{ Clinical features: } \\
\hline Age/sex & $39 \mathrm{~F}$ & $49 M$ & $52 M$ & $\begin{array}{l}17-57(\text { mean } 34) \\
M: F=7: 3\end{array}$ \\
\hline Preceding infection & URTI & C Jejuni enteritis & URTI & $10 / 10$ \\
\hline Symptoms at onset & Bulbar & Bulbar and sensory & Bulbar and sensory & $\begin{array}{l}6 / 10 \text { ocular } \\
5 / 10 \text { ataxia } \\
5 / 10 \text { sensory }\end{array}$ \\
\hline Dysarthria & ++ & ++ & ++ & $9 / 10$ \\
\hline Dysphagia & ++ & ++ & $++t$ & $9 / 10$ \\
\hline Perioral paraesthaesia & - & - & + & $5 / 10$ \\
\hline Facial weakness & - & - & ++ & $9 / 10$ \\
\hline Ptosis & - & - & - & $7 / 10$ \\
\hline Ophthalmoplegia & - & - & - & $10 / 10$ \\
\hline Ataxia & - & + & - & $10 / 10$ \\
\hline Areflexia & Partial & Complete & Complete & $10 / 10$ \\
\hline Limp paraesthesiae & Distal & Distal & - & $5 / 10$ \\
\hline $\begin{array}{l}\text { Limb weakness } \\
\text { (MRC grade) }\end{array}$ & No & No & $\begin{array}{l}\text { Yes } \\
(4)\end{array}$ & $\begin{array}{l}6 / 10 \text { No } \\
4 / 10 \text { grade } 4\end{array}$ \\
\hline Ventilated & No & No & No & $1 / 10$ (4 days) \\
\hline Tube fed & No & No & Yes & $3 / 10$ \\
\hline Specific treatment & None & None & $\mathrm{HIg}$ & $\begin{array}{l}9 / 10 \\
(6 \times \mathrm{HIg}, 3 \times \mathrm{PE})\end{array}$ \\
\hline
\end{tabular}

URTI = Upper respiratory tract infection; HIg = human immune globulin; PE = plasma exchange; $+=$ mild; $++=$ moderate; $+++=$ severe; $-=$ absent.

assays were performed with a panel of gangliosides comprising GM1, GM2, GM3, GD1a, GD1b, GD3, GT1a, GT1b, and GQ1b in a standard enzyme linked immunosorbent assay (ELISA), and a thin layer chromatography (TLC) overlay technique as previously described. ${ }^{9}$ Titres were calculated by end point dilution analysis. Our normal ranges for antiganglioside IgG antibodies are set at $<1 / 500$ (2 SD above the mean of a panel of normal and disease control serum samples). IgG subclass analysis was performed as previously described. ${ }^{10}$

For statistical analysis the antibody titres were $\log _{2}$ transformed. Unpaired Student's $t$ test was then used to assess the significance of the differences of the titres of anti-GQ1b and anti-GT1a antibodies between the group of three oropharyngeal patients and the 10 patients with Miller Fisher syndrome. The ratios (GQ1b:GT1a and GT1a:GQ1b) of the log transformed titres were estimated for each individual patient and the Mann-Whitney $U$ test was used to assess the differences in the ratios between the two groups.

\section{Results}

IgG antibodies to GQ1b and GT1a gangliosides were found in high titres in all three patients (table 2). Reactivities found by ELISA were confirmed using TLC overlay (not shown). Raised antibodies to GD3 (two

Table 2 Laboratory features of the three oropharyngeal patients and the 10 Miller Fisher patients

\begin{tabular}{|c|c|c|c|c|}
\hline & Patient 1 & Patient 2 & Patient 3 & $10 M F S$ patients \\
\hline $\begin{array}{l}\text { CSF protein }(\mathrm{g} / \mathrm{l}) \\
\text { (NR } 0.1-0.5 \mathrm{~g} / \mathrm{l})\end{array}$ & 0.44 & 0.55 & 0.86 & $7 / 10^{\star}(0.64)$ \\
\hline $\begin{array}{l}\text { IgG antiganglioside } \\
\text { (upper limit of no } \\
\text { GQ1b } \\
\text { GT1a } \\
\text { GT1b } \\
\text { GD1a } \\
\text { GD1b } \\
\text { GD3 } \\
\text { GM1 }\end{array}$ & $\begin{array}{l}\text { res } \\
\text { nal }=1 / 500 \\
1 / 3100 \\
1 / 2900 \\
- \\
- \\
\overline{1 / 480}\end{array}$ & $\begin{array}{l}+ \text { ve (mean } \\
1 / 2600 \\
1 / 7600 \\
- \\
- \\
1 / 240 \\
- \\
-\end{array}$ & $\begin{array}{l}1 / 1500 \\
1 / 12500 \\
- \\
- \\
- \\
1 / 570\end{array}$ & $\begin{array}{l}10 / 10(1 / 8000) \\
9 / 10(1 / 5000) \\
6 / 10(1 / 750) \\
0 / 10- \\
2 / 10(1 / 1000) \\
6 / 10(1 / 500) \\
0 / 10-\end{array}$ \\
\hline
\end{tabular}

${ }^{\star} \mathrm{CSF}$ examined in $7 / 10$ patients; - = negative. patients) or GDlb (one patient) were also present but remained within the normal range. No patients had detectable antibodies to GT1b, GM1, or to the other gangliosides tested. IgG subclass analysis showed that both the anti-GQ1b and anti-GT1a IgG antibody reactivities resided in the $\operatorname{IgG} 3$ subclass in patients 1 and 2 , and in the IgG1 subclass in patient 3 (data not shown).

All 10 patients with Miller Fisher syndrome had raised antibody titres (table 2) to GQ1b and nine had raised GT1a antibodies. The patient with Miller Fisher syndrome with a low GT1 a titre $(1 / 220)$ had clearly raised titres to GQ1b (1/2600) and to GT1b (1/3200). Many of the patients with Miller Fisher syndrome and oropharyngeal patients also had detectable antibody titres to structurally related gangliosides such as GD1b, GT1b, and GD3 (table 2). Although these titres were below $\mathrm{I} / 500$ in many instances and would not be considered significant in isolation, the fact that they occurred in the presence of high titres to GT1a and GQ1b does suggest that they are a significant element of this acute immune response.

In the oropharyngeal patients the mean antibody titres to GT1a (1/6500) were higher than those to GQ1b (1/2250); by contrast with the patients with Miller Fisher syndrome the mean GT1a titre $(1 / 5000)$ was lower than the mean GQ1b titre (1/8000). However, statistical analysis of the titres to either antigen showed no significant differences between the patients with oropharyngeal palsy and patients with Miller Fisher syndrome $(P=0.19$ for GQ1b; $P=0.78$ for GT1a). Similarly analysis of the ratios of the paired values between both groups just failed to achieve significance at the $5 \%$ level $(0 \cdot 05<\mathrm{P}<0 \cdot 10)$.

\section{Discussion}

The oropharyngeal variant of Guillain-Barré syndrome may occur in isolation but usually occurs as a transitional syndrome with other features of either Guillain-Barré syndrome or Miller Fisher syndrome. Although these three patients may fit into a transitional category as 
each one had minor symptoms or signs elsewhere, the major clinical manifestations define a clearly identifiable phenotype. None of these patients closely resemble Ropper's description of pharyngeal-cervical-brachial Guillain-Barré syndrome in that all had sensory symptoms, none had ptosis, and proximal upper limb weakness was mild if present. ${ }^{1}$ The most striking clinical feature of these patients was the absence of ophthalmoplegia and ptosis, particularly when other features of Miller Fisher syndrome were present, such as in patient 3 . These patients clearly show that the antiGQ1b antibody need not necessarily be associated with clinically evident ophthalmoplegia and that these antibodies should be sought in any patient with acute craniopharyngeal palsy of unknown cause.

The clinical expression of anti-GQ1b antibody associated syndromes may show geographic variation: in their series of 19 and six patients with Miller Fisher syndrome, Chiba et $a l^{7}$ and Yuki et $a l^{4}$ reported oropharyngeal weakness as minimal or absent. By contrast, nine of the last 10 consecutive anti-GQ1b antibody positive patients with Miller Fisher syndrome admitted to our institute had symptomatic oropharyngeal weakness which in three patients was severe enough to require tube feeding or mechanical ventilation. All 10 patients exhibited the clinical triad of ophthalmoplegia, ataxia, and areflexia without limb weakness. Thus our local experience is that the motor manifestations of Miller Fisher syndrome are not restricted to the extraocular muscles but invariably involve the oropharynx. This does not seem to be the pattern of illness seen in Japan despite similar antiganglioside antibody serology. The explanation(s) for these geographic variations is unknown but may include racial differences in the ganglioside content of different cranial nerves, immunogenetic variations in the host, or structural variations in the provoking antigen(s). Alternatively this may simply represent bias in case ascertainment and reporting.

The fine specificity of the antiganglioside antibodies, as assessed by relative titres to GQ1b and GT1a and reactivity with other structurally similar disialosyl containing gangliosides such as GD1b, GD3, and GT1b, is compatible with many previous studies. ${ }^{3-5-10}$ The IgG subclass data suggest that the population of antibiodies reacting with GQ1b is also reacting with GT1a and vice versa, although this has not been formally established by cross absorption studies. The difference in relative titres to GQ1b and GT1a may be accounted for by a partial preference for one or other ganglioside and is consistent with their very close structural similarity. The absence of reactivity with GT1b indicates that a terminal disialylated galactose (common to both GQ1b and GT1a but absent on GT1b) is an essential feature of the reactive epitope. The relative reactivity with GQ1b and GT1a does not statistically correlate with the clinical pattern of craniopharyngeal involvement in the Miller Fisher syndrome or oropharyngeal patient groups. However, the data tend towards the GT1a/GQ1b titre ratio being higher in the oropharyngeal group than in the Miller Fisher syndrome group, suggesting that anti-GQ1b and anti-GT1a antibodies may be markers for ophthalmoplegia and pharyngeal palsy respectively. In addition, the presence of anti-GD1b or anti-GD3 antibodies seems to be associated with more prominent sensory involvement, as previously suggested." The few patients studied preclude detailed statistical analysis but these concepts are compatible with previously published data. ${ }^{8-11}$ Although the relative amount of GQ1b in human oculomotor nerves compared with peripheral nerve has been analysed and found to be high, ${ }^{7}$ its quantitative relation with GT1a and other disialylated gangliosides throughout the craniobulbar region is unknown.

We are grateful to Dr Peter Harvey for allowing us to report patient 3 .

This work has been funded by the Guillain-Barre Syndrome Support Group of Great Britain and the Wellcome Trust.

1 Ropper AH. Miller Fisher syndrome and other acute variants of Guillain-Barré syndrome. In: McLeod AG, ed. Inflammatory neuropathies. London: Balliére Tindall, 994:95-106.

2 Fisher $M$. An unusual variant of acute idiopathic polyneuritis (syndrome of ophthalmoplegia, ataxia and areflexia). $N$ Engl f Med 1956;255:57-65

3 Chiba A, Kusunoki S, Shimizu T, Kanazawa I. Serum IgG antibody to ganglioside GQIb is a possible marker of Miller Fisher syndrome. Ann Neurol 1992;31:677-9.

4 Yuki N, Sato S, Tsuji S, Ohsawa T, Miyatake T. Frequent presence of anti-GO1b antibody in Fisher's syndrome. Neurology 1993;43:414-7.

5 Willison HJ, Veitch J, Paterson G, Kennedy PGE. Miller Fisher syndrome is associated with serum antibodies to GQ1b ganglioside. $\mathcal{F}$ Neurol Neurosurg Psychiatry 1993; 56:204-6.

6 Dahaene I, Martin K, Geens K, Cras P. Guillain-Barré syndrome with ophthalmoplegia: clinicopathologic study of the central and peripheral nervous systems, including the oculomotor nerves. Neurology 1986;36:851-4.

7 Chiba A, Kusonoki S, Obata H, Machinami R, Kanazawa I Serum anti-GQ1b IgG antibody is associated with ophthalmoplegia in Miller Fisher syndrome and GuillainBarré syndrome: clinical and immunohistochemical studies. Neurology 1993;43:1911-7.

8 Mizoguchi K, Hase A, Obi T, et al. Two species of antiganglioside antibodies in a patient with a pharyngealcervical-brachial variant of Guillain-Barré syndrome. $\mathcal{f}$ Neurol 1994;57:1121-3.

9 Willison HJ, Paterson G, Veitch J, Inglis G, Barnett S Peripheral neuropathy associated with monoclonal IgM Peripheral neuropathy associated with monoclonal IgM
anti-Pr2 cold agglutinins. $\mathcal{F}$ Neurol Neurosurg Psychiatry anti-Pr2 cold agg

10 Willison HJ, Veitch J. Immunoglobulin subclass distribution and binding characteristics of anti-GQ1b antibodies in Miller Fisher syndrome. $\mathcal{F}$ Neuroimmunol 1994;50 159-65.

11 Willison HJ, Almemar A, Veitch J, Thrush D. Acute ataxic neuropathy with cross-reactive antibodies to GD1b and GD3 gangliosides. Neurology 1994;44:2395-7. 